\title{
Synthesis of Some Pyrazole Containing Chalcones and Pyridine-3-Carbonitriles and Study of their Anti-inflammatory Activity
}

\author{
ANIL G. GADHAVE* and BHAGWAT K UPHADE \\ P. G. Department of Chemistry and Research Centre, \\ Padmashri Vikhe Patil College, Pravaranagar, Dist-Ahmednagar-413713, India. \\ ${ }^{*}$ Corresponding author E-mail : anilgadhave @ gmail.com \\ http://dx.doi.org/10.13005/ojc/330125
}

(Received: October 13, 2016; Accepted: January 01, 2017)

\begin{abstract}
In present work the 4-formyl pyrazole (1) were treated with 4-morpholinoacetophene (2) in the presence of aqueous $\mathrm{KOH}$ to give Chalcones (3). The chalcones are subjected to react with malononitrile using sodium methoxide as a base in methanol to give pyridine-3-carbonitriles (4). The synthesized chalcone and pyridine-3-carbonitrile derivatives were subjected for study of their antiinflammatory activity by the well known carrageenan induced rat paw edema method. The products $3 a, 3 e$ and $4 \mathrm{e}$ exhibited good activity as compared with standard diclofenac drug. The formation of all compounds was established by the spectral techniques such as Infra Red, ${ }^{1} \mathrm{H}-\mathrm{NMR}$ and mass.
\end{abstract}

Keywords: Anti-inflammatory, Pyrazole, Pridine-3-carbonitirle, Chalcone.

\section{INTRODUCTION}

In the last few decades research has progressed in understanding the mechanism of inflammation which is a physiological process essential for the survival but it can also cause human morbidity and mortality ${ }^{1,2}$. The disorders related to pain cannot be easily identified or removed. The current available drugs which possess antiinflammatory activity are known to have some boundaries in medical use that are ulceration and gastrointestinal haemorrhage, addiction and acceptance for opiates ${ }^{3}$. These limitations creates some challenges for organic and medicinal chemist to design and develop more useful antiinflammatory drugs ${ }^{4,5}$. The efforts for improving the pharmacological profile of anti-inflammatory agents led towards discovery of multiple isoform of cyclooxygenase $(\mathrm{COX})^{6}$. 
Chalcones are the biologically active scaffold used in synthesis of various heterocyclic compounds. They are also known to possess pharmacological activites such as tyrosine inhibition ${ }^{7}$, anti-inflammatory ${ }^{8}$, anticancer ${ }^{9}$, antioxidant ${ }^{10}$ and nitric oxide inhibition ${ }^{11}$. The incorporation of pyrazole moiety into heterocyclic compounds gave the pharmaceutical drugs such as celecoxib ${ }^{12}$ and rimonabant ${ }^{13}$. They also possess biological activites such as anti-inflammatory ${ }^{14}$, A3 adenosine receptor antagonist ${ }^{15}$, antiviral ${ }^{16}$ and antifungal ${ }^{17}$. The $\mathrm{N}$-functionalized morpholine containing heterocyclic compounds are biologically important motifs which are known to possess antidiabetic ${ }^{18}$, antiemetic ${ }^{19}$, anti-inflammatory and central nervous system ${ }^{20}$ activities.

The pyridine is a heterocyclic compound found in variety of natural and synthetic products possessing pharmacological activities such as hypoglycemic, dopamine transporter inhibitors and anti-inflammatory ${ }^{21,22}$. They also have application in dyes for synthetic fabrics ${ }^{23}$, light emitting ${ }^{24}$ and electroluminescence ${ }^{25}$ devices.

The biological applications of chalcone, pyridine, pyrazole, morpholine and in continuation to our ongoing research ${ }^{26-29}$ herein we report the synthesis of chalcones, pyridine-3-carbonitriles and their anti-inflammatory activity.

\section{MATERIALS AND METHODS}

The chemicals used were of analytical grade and are obtained commercially. The M. P. of all products was performed in open capillaries. The Shimadzu FTIR spectrophotometer was used for the Infra Red Spectrum (IR) using $\mathrm{KBr}$ and only functional group frequencies are given in $\mathrm{cm}^{-1}$. The Proton Magnetic Resonance (PMR) spectrum was carried over Bruker Avance spectrometer ( $400 \mathrm{MHz}$ ) using TMS as an internal standard and $\mathrm{CDCl}_{3}$ solvent. The Finnigan mass spectrometer was used for Mass spectrum. The TLC plates available commercially were used to check the progress and completion of reactions.

General protocol for synthesis of 1,3-disubstituted prop-2-en-1-one (chalcones): (3a-e)

The 4-formyl pyrazole $(10 \mathrm{mmol})$ and 4-morpholinoacetophenone $(10 \mathrm{mmol})$ were taken in R. B. flask and to it $20 \mathrm{ml}$ of EtOH was added. To the same flask $10 \mathrm{ml}$ of $20 \% \mathrm{KOH}$ solution was added. The resulting reaction content was allowed to attain room temperature and stirred for $24 \mathrm{~h}$. The progress and completion of reaction was monitored by TLC and then the reaction contents were added in ice and neutralized by acetic acid. The precipitated product was separated by filtration and dried. The product was purified by recrystallization using ethanol to furnish chalcones (3a-e). The characterization data of synthesized compounds is as follows.

\section{Compound $3 \mathbf{a}$}

Yield $70 \%$, m.p. $282-283^{\circ} \mathrm{C}$; IR $\left(\mathrm{KBr}, \mathrm{cm}^{-1}\right)$ : 1688 (Carbonyl), $1654(\mathrm{C}=\mathrm{N}), 1625(\mathrm{C}=\mathrm{C}), 1235$ (C-O); ${ }^{1} \mathrm{H}$ NMR $\left(\mathrm{CDCl}_{3}, \delta\right): 3.35$ (triplet, $4 \mathrm{H},-\mathrm{N}-\mathrm{CH}_{2}-$ ), 3.70 (triplet, $4 \mathrm{H},-\mathrm{O}-\mathrm{CH}_{2}-$ ), 6.94 (doublet, $2 \mathrm{H}, \mathrm{J}=8 \mathrm{~Hz}$, $\mathrm{H}$-Aromatic), 7.31-7.87 (multiplet, 12H, H-Aromatic \& $\mathrm{CH}=\mathrm{CH}), 7.95$ (d, 2H, J=8 Hz, H-Aromatic), 8.22 (s, $1 \mathrm{H}$, proton of pyrazole ring); Mass m/z $435[\mathrm{M}]^{+}$.

\section{Compound 3b}

Yield 77\%, m.p. $252-253^{\circ} \mathrm{C}$; IR $\left(\mathrm{KBr}, \mathrm{cm}^{-1}\right)$ : 1690 (Carbonyl), $1648(\mathrm{C}=\mathrm{N}), 1627(\mathrm{C}=\mathrm{C}), 1230$ (C-O); ${ }^{1} \mathrm{H}$ NMR $\left(\mathrm{CDCl}_{3}, \delta\right): 2.17$ (singlet, $3 \mathrm{H}, \mathrm{CH}_{3}$ of Aromatic ring), 3.45 (triplet, $4 \mathrm{H},-\mathrm{N}-\mathrm{CH}_{2}-$ ), 3.90 (triplet, $4 \mathrm{H},-\mathrm{O}-\mathrm{CH}_{2}-$ ), 7.08 (doublet, $2 \mathrm{H}, \mathrm{J}=8 \mathrm{~Hz}$, $\mathrm{H}$-Aromatic), 7.28-7.98 (multiplet, $11 \mathrm{H}, \mathrm{H}$-Aromatic \& $\mathrm{CH}=\mathrm{CH}$ ), 8.01 (doublet, $2 \mathrm{H}, \mathrm{J}=8 \mathrm{~Hz}, \mathrm{H}$-Aromatic), 8.35 (singlet, $1 \mathrm{H}$, proton of pyrazole ring); Mass $\mathrm{m} / \mathrm{z}$ $449[\mathrm{M}]^{+}$.

\section{Compound $3 \mathrm{c}$}

Yield $73 \%$, m.p. $220-221^{\circ} \mathrm{C}$; IR ( $\mathrm{KBr}$, $\left.\mathrm{cm}^{-1}\right)$ : 1685 (Carbonyl), $1652(\mathrm{C}=\mathrm{N}), 1620(\mathrm{C}=\mathrm{C})$, 1222 (C-O), 1090 (Ar-Cl); ${ }^{1} \mathrm{H}$ NMR $\left(\mathrm{CDCl}_{3}, \delta\right): 3.32$ (triplet, $4 \mathrm{H},-\mathrm{N}-\mathrm{CH}_{2}-$ ), 3.83 (triplet, $4 \mathrm{H},-\mathrm{O}-\mathrm{CH}_{2}-$ ), 6.91 (doublet, $2 \mathrm{H}, \mathrm{J}=8 \mathrm{~Hz}, \mathrm{H}$-Aromatic), 7.34-7.84 (multiplet, 11H, $\mathrm{H}$-Aromatic \& $\mathrm{CH}=\mathrm{CH}$ ), 7.94 (doublet, $2 \mathrm{H}, \mathrm{J}=8 \mathrm{~Hz}, \mathrm{H}$-Aromatic), 8.33 (singlet, $1 \mathrm{H}$, proton of pyrazole ring); Mass m/z $469[\mathrm{M}]^{+}$.

\section{Compound 3d}

Yield $69 \%$, m.p. $230-231^{\circ} \mathrm{C}$; IR $(\mathrm{KBr}$, $\left.\mathrm{cm}^{-1}\right)$ : 1687 (Carbonyl), $1649(\mathrm{C}=\mathrm{N}), 1632(\mathrm{C}=\mathrm{C})$, 1244 (C-O), 1188 (Ar-F); ${ }^{1} \mathrm{H}$ NMR $\left(\mathrm{CDCl}_{3}, \delta\right): 3.32$ (triplet, $4 \mathrm{H},-\mathrm{N}-\mathrm{CH}_{2}-$ ), 3.87 (triplet, $4 \mathrm{H},-\mathrm{O}-\mathrm{CH}_{2}-$ ), 6.89 (doublet, $2 \mathrm{H}, \mathrm{J}=8 \mathrm{~Hz}, \mathrm{H}$-Aromatic), 7.16-7.88 (multiplet, $11 \mathrm{H}, \mathrm{H}$-Aromatic \& $\mathrm{CH}=\mathrm{CH}$ ), 7.93 (doublet, 
$2 \mathrm{H}, \mathrm{J}=8 \mathrm{~Hz}, \mathrm{H}$-Aromatic), 8.33 (singlet, $1 \mathrm{H}$, proton of pyrazole ring); Mass m/z 453 [M] .

\section{Compound $3 \mathrm{e}$}

Yield $60 \%$, m.p. $238-240^{\circ} \mathrm{C}$; IR $\left(\mathrm{KBr}, \mathrm{cm}^{-1}\right)$ : 1680 (Carbonyl), $1653(\mathrm{C}=\mathrm{N}), 1619(\mathrm{C}=\mathrm{C}), 1216$ (C-O); ${ }^{1} \mathrm{H} \mathrm{NMR}\left(\mathrm{CDCl}_{3}, \delta\right): 3.31$ (triplet, $4 \mathrm{H},-\mathrm{N}-\mathrm{CH}_{2}-$ ), 3.84 (multiplet, $4 \mathrm{H}$ of $-\mathrm{O}-\mathrm{CH}_{2}-\& 3 \mathrm{H}$ of Aromaticmethoxy group proton), 6.90 (doublet, $2 \mathrm{H}, \mathrm{J}=8 \mathrm{~Hz}$, $\mathrm{H}$-Aromatic), 7.00-7.89 (multiplet, $11 \mathrm{H}, \mathrm{H}$-Aromatic \& $\mathrm{CH}=\mathrm{CH}$ ), 7.95 (doublet, $2 \mathrm{H}, \mathrm{J}=8 \mathrm{~Hz}, \mathrm{H}$-Aromatic), 8.32 (singlet, $1 \mathrm{H}$, proton of pyrazole ring); Mass $\mathrm{m} / \mathrm{z}$ $465[\mathrm{M}]^{+}$.

\section{General protocol for synthesis of pyridine-3- carbonitriles (4a-e)}

The chalcone 3 ( $1 \mathrm{mmol}$ ) and malononitrile (1 mmol) was taken in methanol $(10 \mathrm{~mL})$ in a R. B. flask. To this sodium methoxide was added (prepared freshly from sodium metal and absolute methanol) and the reaction contents were refluxed for 3-4 hours. The progress and completion of reaction was checked by TLC. Then the reaction contents were allowed to attain the room temperature.
The precipitated product was filtered, washed with water and dried. The products were purified by recrystallization using ethanol to furnish pure pyridine-3-carbonitrile derivatives (4a-e). The characterization data of synthesized compounds is as follows.

\section{Compound 4a}

Yield $81 \%$, m.p. $259-260^{\circ} \mathrm{C}$; IR $\left(\mathrm{KBr}, \mathrm{cm}^{-1}\right)$ : $2230(\mathrm{CN}), 1660(\mathrm{C}=\mathrm{N}), 1601(\mathrm{C}=\mathrm{C}), 1235(\mathrm{C}-\mathrm{O})$; ${ }^{1} \mathrm{H}$ NMR $\left(\mathrm{CDCl}_{3}, \delta\right): 3.32$ (triplet, $\left.4 \mathrm{H},-\mathrm{N}-\mathrm{CH}_{2}-\right)$, 3.86 (triplet, $4 \mathrm{H}$ of $-\mathrm{O}-\mathrm{CH}_{2}-$ ), 4.17 (singlet, $3 \mathrm{H}$, Aromaticmethoxy group proton), 6.89 (doublet, $2 \mathrm{H}, \mathrm{J}=8 \mathrm{~Hz}$, $\mathrm{H}$-Aromatic), 7.34-7.90 (multiplet, 11H, H-Aromatic), 7.93 (doublet, $2 \mathrm{H}, \mathrm{J}=8 \mathrm{~Hz}, \mathrm{H}$-Aromatic), 8.52 (singlet, $1 \mathrm{H}$, proton of pyrazole ring); Mass m/z 513 [M] .

\section{Compound 4b}

Yield $78 \%$, m.p. $286-287^{\circ} \mathrm{C}$; IR $\left(\mathrm{KBr}, \mathrm{cm}^{-1}\right)$ : 2235 (CN), 1647 (C=N), 1595 (C=C), 1241 (C-O); ${ }^{1} \mathrm{H}$ NMR $\left(\mathrm{CDCl}_{3}, \delta\right): 2.39$ (singlet, $3 \mathrm{H}, \mathrm{CH}_{3}$ of Aromatic ring), 3.25 (triplet, $4 \mathrm{H},-\mathrm{N}-\mathrm{CH}_{2}-$ ), 3.88 (triplet, $4 \mathrm{H}$ of -O- $\mathrm{CH}_{2}-$ ), 4.17 (singlet, $3 \mathrm{H}$, Aromatic-methoxy group proton), 6.92 (doublet, $2 \mathrm{H}, \mathrm{J}=8 \mathrm{~Hz}, \mathrm{H}$-Aromatic), 7.12

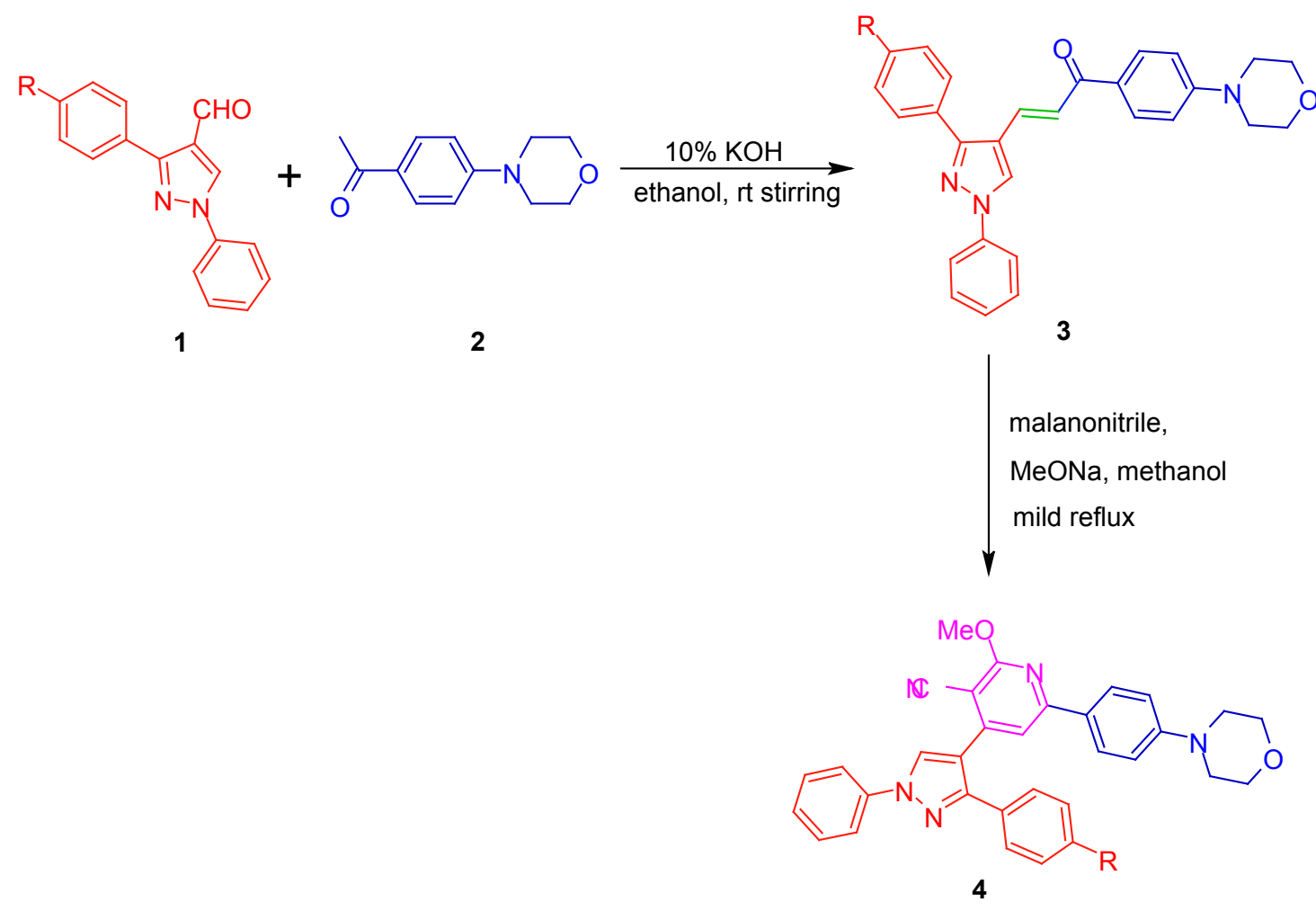

Scheme 
(singlet, 1H, H-Aromatic), 7.19-7.73 (multiplet, 9H, $\mathrm{H}$-Aromatic), 7.79 (doublet, $2 \mathrm{H}, \mathrm{J}=8 \mathrm{~Hz}, \mathrm{H}$-Aromatic), 8.48 (singlet, $1 \mathrm{H}$, proton of pyrazole ring); Mass $\mathrm{m} / \mathrm{z}$ $527[\mathrm{M}]^{+}$.

\section{Compound 4c}

Yield $82 \%$, m.p. $262-263^{\circ} \mathrm{C}$; IR $\left(\mathrm{KBr}, \mathrm{cm}^{-1}\right)$ : $2216(\mathrm{CN}), 1657(\mathrm{C}=\mathrm{N}), 1599(\mathrm{C}=\mathrm{C}), 1255(\mathrm{C}-\mathrm{O})$, 1075 (Ar-Cl); ${ }^{1} \mathrm{H}$ NMR $\left(\mathrm{CDCl}_{3}, \delta\right): 3.29$ (triplet, $4 \mathrm{H},-\mathrm{N}-\mathrm{CH}_{2}-$ ), 3.92 (triplet, $4 \mathrm{H}$ of $-\mathrm{O}-\mathrm{CH}_{2}-$ ), 4.18 (singlet, $3 \mathrm{H}$, Aromatic-methoxy group proton), 7.02 (doublet, 2H, J=8 Hz, H-Aromatic), 7.11 (singlet, $1 \mathrm{H}$, $\mathrm{H}$-Aromatic), 7.35-7.81 (multiplet, 11H, H-Aromatic), 8.46 (singlet, $1 \mathrm{H}$, proton of pyrazole ring); Mass $\mathrm{m} / \mathrm{z}$ $548[\mathrm{M}]^{+}$.

\section{Compound 4d}

Yield $75 \%$, m.p. $258-259^{\circ} \mathrm{C}$; IR $(\mathrm{KBr}$, $\left.\mathrm{cm}^{-1}\right): 2221(\mathrm{CN}), 1650(\mathrm{C}=\mathrm{N}), 1605(\mathrm{C}=\mathrm{C}), 1232$ (C-O), 1126 (Ar-F); ${ }^{1} \mathrm{H}$ NMR $\left(\mathrm{CDCl}_{3}, \delta\right): 3.25$ (triplet, $4 \mathrm{H},-\mathrm{N}-\mathrm{CH}_{2}-$ ), 3.86 (triplet, $4 \mathrm{H}$ of $-\mathrm{O}-\mathrm{CH}_{2}-$ ), 4.18 (singlet, $3 \mathrm{H}$, Aromatic-methoxy group proton), 6.90

Table 1: The physical data of synthesized chalcones and pyridine-3-carbonitriles

\begin{tabular}{cccccc}
\hline Comp. & $\begin{array}{c}\mathbf{R} \\
\text { Group }\end{array}$ & $\begin{array}{c}\text { Molecular } \\
\text { Formula }\end{array}$ & $\begin{array}{c}\text { Molecular } \\
\text { Weight (gm) }\end{array}$ & $\begin{array}{c}\text { Melting } \\
\text { Point }\left({ }^{\circ} \mathbf{C}\right)\end{array}$ & $\begin{array}{c}\text { Yield } \\
\text { (\%) }\end{array}$ \\
\hline $3 a$ & $\mathrm{H}$ & $\mathrm{C}_{28} \mathrm{H}_{25} \mathrm{~N}_{3} \mathrm{O}_{2}$ & 435.51 & $282-283$ & 70 \\
$3 \mathrm{~b}$ & $4-\mathrm{Me}$ & $\mathrm{C}_{29} \mathrm{H}_{27} \mathrm{~N}_{3} \mathrm{O}_{2}$ & 449.54 & $252-253$ & 77 \\
$3 \mathrm{c}$ & $4-\mathrm{Cl}$ & $\mathrm{C}_{28} \mathrm{H}_{24} \mathrm{CIN}_{3} \mathrm{O}_{2}$ & 469.96 & $220-221$ & 73 \\
$3 \mathrm{~d}$ & $4-\mathrm{F}$ & $\mathrm{C}_{28} \mathrm{H}_{24} \mathrm{FN}_{3} \mathrm{O}_{2}$ & 453.50 & $230-231$ & 69 \\
$3 e$ & $4-\mathrm{Me}$ & $\mathrm{C}_{29} \mathrm{H}_{27} \mathrm{~N}_{3} \mathrm{O}_{3}$ & 465.54 & $238-239$ & 60 \\
$4 \mathrm{a}$ & $\mathrm{H}$ & $\mathrm{C}_{32} \mathrm{H}_{27} \mathrm{~N}_{5} \mathrm{O}_{2}$ & 513.58 & $259-260$ & 81 \\
$4 \mathrm{~b}$ & $4-\mathrm{Me}$ & $\mathrm{C}_{33} \mathrm{H}_{29} \mathrm{~N}_{5} \mathrm{O}_{2}$ & 527.61 & $286-287$ & 78 \\
$4 \mathrm{c}$ & $4-\mathrm{Cl}$ & $\mathrm{C}_{32} \mathrm{H}_{26} \mathrm{CIN}_{5} \mathrm{O}_{2}$ & 548.03 & $262-263$ & 82 \\
$4 \mathrm{~d}$ & $4-\mathrm{F}$ & $\mathrm{C}_{32} \mathrm{H}_{26} \mathrm{FN}_{5} \mathrm{O}_{2}$ & 531.57 & $258-259$ & 75 \\
$4 \mathrm{e}$ & $4-\mathrm{Me}$ & $\mathrm{C}_{33} \mathrm{H}_{29} \mathrm{~N}_{5} \mathrm{O}_{3}$ & 543.61 & $260-261$ & 71 \\
\hline
\end{tabular}

Table 2: The anti-inflammatory activity study results of chalcones and pyridine-3-carbonitriles

\begin{tabular}{lcccc}
\hline Compound & $\mathbf{R}$ & $\begin{array}{c}\text { Control } \\
\text { (paw volume } \\
\text { in mm) }\end{array}$ & $\begin{array}{c}\text { Test } \\
\text { (paw volume } \\
\text { in mm) }\end{array}$ & Difference \\
\hline Control & - & 9.8 & - & - \\
Diclofenac & - & 9.8 & 8.1 & 1.7 \\
3a & $\mathrm{H}$ & 9.8 & 8.2 & 1.6 \\
3b & $4-\mathrm{Me}$ & 9.8 & 9.1 & 0.7 \\
3c & $4-\mathrm{Cl}$ & 9.8 & 9.2 & 0.6 \\
3d & $4-\mathrm{F}$ & 9.8 & 9.1 & 0.7 \\
3e & $4-\mathrm{OMe}$ & 9.8 & 7.8 & 2.0 \\
$4 \mathrm{a}$ & $\mathrm{H}$ & 9.8 & 9.3 & 0.5 \\
4b & $4-\mathrm{Me}$ & 9.8 & 9.2 & 0.6 \\
$4 \mathrm{c}$ & $4-\mathrm{Cl}$ & 9.8 & 9.2 & 0.6 \\
$4 \mathrm{~d}$ & $4-\mathrm{F}$ & 9.8 & 9.1 & 0.7 \\
$4 \mathrm{e}$ & $4-\mathrm{OMe}$ & 9.8 & 8.5 & 1.3 \\
\hline
\end{tabular}


(doublet, 2H, J=8 Hz, H-Aromatic), 7.07 (singlet, $1 \mathrm{H}$, $\mathrm{H}$-Aromatic), 7.09-7.81 (multiplet, 11H, $\mathrm{H}$-Aromatic), 8.47 (singlet, $1 \mathrm{H}$, proton of pyrazole ring); Mass $\mathrm{m} / \mathrm{z}$ $531[\mathrm{M}]^{+}$.

\section{Compound $4 \mathrm{e}$}

Yield $71 \%$, m.p. $260-261^{\circ} \mathrm{C}$; IR $\left(\mathrm{KBr}, \mathrm{cm}^{-1}\right)$ : $2215(\mathrm{CN}), 1662(\mathrm{C}=\mathrm{N}), 1610(\mathrm{C}=\mathrm{C}), 1254(\mathrm{C}-\mathrm{O})$; ${ }^{1} \mathrm{H}$ NMR $\left(\mathrm{CDCl}_{3}, \delta\right): 3.24$ (triplet, $\left.4 \mathrm{H},-\mathrm{N}-\mathrm{CH}_{2}-\right), 3.82$ (triplet, $4 \mathrm{H}$ of $-\mathrm{O}-\mathrm{CH}_{2}-$ ), 3.95 (singlet, $3 \mathrm{H}$, Aromaticmethoxy group proton), 4.19 (singlet, $3 \mathrm{H}$, Aromaticmethoxy group proton), 6.99 (doublet, $2 \mathrm{H}, \mathrm{J}=8 \mathrm{~Hz}$, $\mathrm{H}$-Aromatic), 7.10 (singlet, 1H, H-Aromatic), 7.317.82 (multiplet, 11H, H-Aromatic), 8.49 (singlet, $1 \mathrm{H}$, proton of pyrazole ring); Mass m/z $543[\mathrm{M}]^{+}$.

\section{Anti-inflammatory activity study}

The chalcones and pyridine-3-carbonitriles were subjected for anti-inflammatory activity study by literature known protocol using carrageenan-induced paw edema test in rats. The study was carried using wistar rats of 150-200 g weight. Standard pellet diet and water ad libitum was fed to the study animals. The $12 \mathrm{~h}$ light and $12 \mathrm{~h}$ dark cycle at $25^{\circ} \mathrm{C}$ was maintained for the animals. Animal experiment was approved by IAEC/ CPCSEA. The overnight fasted rats were grouped in three animals per group. The control group was given vehicle (1\% CMC in water in a volume of $10 \mathrm{~mL} / \mathrm{kg}$ ) only. In vehicle $(10 \mathrm{~mL} / \mathrm{kg})$ the Diclofenac $(10 \mathrm{mg} / \mathrm{kg})$ and test samples $(10 \mathrm{mg} / \mathrm{kg}$ ) was suspended and administered orally to the respective groups. After half hour, 0.1 $\mathrm{ml}$ of $1 \%$ carrageenan solution (prepared in normal saline) was injected in the subplanter region of right hind paw of all animals. After 3 hours, with the help of digital vernier caliper (Mitutoyo, Japan) the edema formed in the paw was measured. Edema induced by carrageenan alone was considered as $100 \%$ induction. Any major decrease in paw volume compared to control group was measured as antiinflammatory response. The outcome obtained during the study is presented in Table 2.

\section{RESULTS AND DISCUSSION}

The synthesis of chalcones and pyridine3-carbonitrile derivatives are shown in scheme 1. The chalcones 3a-e is synthesized by well known Claisen-Schmidt condensation of 4-formyl pyrazole 1a-e with 4-morpholinoacetophenone 2 in presence
$\mathrm{KOH}$ base and ethanol as a solvent under room temperature stirring. The formation of chalcones 3a-e was confirmed on the basis of the spectral techniques. The Infra Red spectrum of chalcone $3 \mathbf{a}$ show characteristic stretching frequencies at 1688 $\mathrm{cm}^{-1}$ for $\mathrm{C}=\mathrm{O}, 1654 \mathrm{~cm}^{-1}$ for $\mathrm{C}=\mathrm{N}, 1625 \mathrm{~cm}^{-1}$ for $\mathrm{C}=\mathrm{C}$ and $1235 \mathrm{~cm}^{-1}$ for $\mathrm{C}-\mathrm{O}$ groups. The ${ }^{1} \mathrm{H}-\mathrm{NMR}$ spectra of compound $3 \mathrm{c}$ shows two triplets at $3.32 \delta$ and $3.83 \delta$ each for four protons are due to $-\mathrm{N}-\mathrm{CH}_{2}-$ and $-\mathrm{O}-\mathrm{CH}_{2}$ - groups of morpholine ring respectively. The aromatic as well as $\mathrm{C}=\mathrm{C}$ group protons appeared in $6.91-7.94 \delta$ while the pyrazole ring proton exhibited singlet at $8.33 \delta$. The mass spectrum of compound $3 e$ shows $\mathrm{m} / \mathrm{z} 465$ peak corresponding to its molecular weight.

The chalcones react with malononitrile in methanol solvent in the presence of freshly prepared sodium methoxide base under reflux condition gave pyridine-3-carbonotriles derivatives $4 a-e$. The formation of pyridine-3-carbonotriles was confirmed on the basis of spectral techniques. The Infra Red spectra of compound $4 \mathrm{~b}$ exhibited peaks at $2235 \mathrm{~cm}^{-1}$ for $\mathrm{CN}$ and $1647 \mathrm{~cm}^{-1}$ due to $\mathrm{C}=\mathrm{N}$ functional groups. The ${ }^{1} \mathrm{H}-\mathrm{NMR}$ spectrum of compound $\mathbf{4} \mathbf{d}$ exhibited two triplets at $3.25 \delta$ and $3.86 \delta$ each for four protons are due to $-\mathrm{N}-\mathrm{CH}_{2}-$ and $-\mathrm{O}-\mathrm{CH}_{2}-$ groups of morpholine ring respectively. The new signal appeared at $4.18 \delta$ as a singlet for three protons is due to $-\mathrm{OCH}_{3}$ group. The aromatic ring protons show multiplet between $6.90-7.81 \delta$. The proton of pyrazole ring shows a singlet at $8.47 \delta$. The mass spectrum of compound $4 \mathrm{e}$ showed $\mathrm{m} / \mathrm{z} 543$ peak corresponding to its molecular weight which confirm its formation.

The anti-inflammatory activity study results are represented in Table no. 2 which reveals that, the compound $3 \mathrm{a}, 3 \mathrm{e}$ and $4 \mathrm{e}$ exhibited good activity as compared with standard diclofenac drug. The other compounds possess moderate activity profile. The good biological activity in chalcone $3 \mathrm{a}$ and $3 \mathrm{e}$ are attributed due to the no substituent in $3 a$ and para methoxy substituent in $3 e$ on phenyl ring of pyrazole moiety. The $4 \mathrm{e}$ pyridine-3-carbonitrile possesses methoxy substituent on phenyl ring of pyrazole which may have enhanced its activity.

Owing to the biological importance associated with pyrazole, morpholine, chalcone and pyridine-3-carbonitriles scaffolds, herein we reports 
the synthesis, characterization and anti-inflammatory activity study of morpholine and pyrazole containing chalcones and pyridine-3-carbonitriles derivatives. The insight obtained in the present study will be useful for development of newer biologically important compounds.

\section{ACKNOWLEDGEMENTS}

Authors are thankful to BCUD, Savitribai
Phule Pune University for financial assistance, The authors are grateful to Pravara Rural Education Society, Pravaranagar (MS) and Principal of our college for providing all necessary facilities. We are also thankful to Prin. Dr. B. K. Karale for the encouragement and valuable guidance. We are also thankful to SAIF, Panjab University, Chandigarh for spectral analysis.

\section{REFERENCES}

1. O'Neill, L. A., Nat. Rev. Drug Discovery, 2006, 5, 549.

2. Cheeseright, T. J.; Holm, M.; Lehmann, F.; Luik, S.; Gottert, M.; Melville, J. L.; Laufer, S., J. Med. Chem., 2009, 52, 4200.

3. Vongtau, H. O.; Abbah, J.; Mosugu, O.; Chindo, B. A.; Ngazal, I. E.; Salawu, A. O.; Kwanashie, H. O.; Gamaniel, K. S., J. Ethnopharmacol.; 2004, 92, 317.

4. Chao, E. Y.; Caravella, J. A.; Watson, M. A.; Campobasso, N.; Ghisletti, S.; Billin, A. N., J. Med. Chem., 2008, 51, 5758.

5. Hynes, J. J.; Dyckmann, A. J.; Lin, S.; Wrobleski, S. T.; Hong, W.; Gillooly, K. M., J. Med. Chem., 2008, 41, 4.

6. Song, Y.; Connor, D. T.; Doubleday, R.; Sorenson, R. J.; Sercel, A. D.; Unangst, P. C.; Roth, B. D.; Gilbersten, R. B.; Chan, K.; Schrier, D. J.; Guglietta, A.; Bornemeier, D. A.; Dyer, R. D., J. Med. Chem., 1999, 42, 1151.

7. Nerya, O.; Musa, R.; Khatib, S.; Tamir, S.; Vaya, J., Phytochemistry, 2004, 65, 1389.

8. Cheng, J. H.; Hung, C. F.; Yang, S. C.; Wang, J. P.; Won, S. J.; Lin, C. N., Bioorg. Med. Chem., 2008, 16, 7270.

9. Lawrence, N. J.; Patterson, R. P.; Ooi, L. L.; Cook, D.; Ducki, S., Bioorg. Med. Chem. Lett., 2006, 16, 5844.

10. Vogel, S.; Ohmayer, S.; Burnner, G.; Heilmann, J., Bioorg. Med. Chem., 2008, 11, 4286.

11. Rojas, J.; Paya, M.; Dominguez J. N.; Ferrandiz, M. L., Bioorg. Med. Chem. Lett., 2002, 12, 1951.

12. Penning, T. D.; Talley, J. J.; Bertenshaw, S.
R.; Carter, J. S.; Collins, P. W.; Docter, S.; Graneto, M. J.; Lee, L. F.; Malecha, J. W.; Miyashiro, J. M.; Rogers, R. S.; Roiger, D. J.; Yu, S. S.; Anderson, G. D.; Burton, E. G.; Cogburn, J. N.; Gregory, S. A.; Koboldt, C. M.; Perkins, W. E.; Seibert, K.; Veenhuizen, A. W.; Zhang, Y. Y.; Isakson, P. C., J. Med. Chem., 1997, 40, 1347.

13. Deng, X.; Mani, N. S., Org. Lett., 2008, 10, 1307.

14. Smith, S. R.; Denhardt, G.; Terminelli, C., Eur. J. Pharmacol., 2001, 432, 107.

15. Baraldi, P. G.; Bovero, A.; Fruttarolo, F.; Romagnoli, R.; Tabrizi, M. A.; Preti, D.; Varani, K.; Borea, P. A.; Moorman, A. R., Bioorg. Med. Chem., 2003, 11, 4161.

16. Baraldi, P. G.; Manfredini, S.; Romagnoli, R.; Stevanato, L.; Zaid, A. N.; Manservigi, R., Nucleosides, Nucleotides Nucleic Acids, 1998, 17, 2165.

17. Prakash, O.; Kumar, R.; Prakash, V., Eur. J. Med. Chem., 2008, 43, 435.

18. Manfred R.; Michael, M.; Robert, S.;Wolfgang, G., Eur. Patent Appl. EP 334146:28, 1989.

19. Hale, J. J.; Mills, S. G.; MacCross, M.; Dorn, C. P.; Finke, P. E.; Budhu, R. J., J. Med. Chem., 2000, 43, 1234.

20. Varma, R. S.; Prakash, C.; Prasad, R., J. Chem. Soc. Pak., 1986, 8, 117.

21. Enyedy, I. J.; Sakamuri, S.; Zaman, W. A.; Johnson, K. M.;Wang, S., Bioorg. Med. Chem. Lett., 2003, 13, 513.

22. Tian, L.; Song, J.; Wang, J.; Liu, B., Chin. Chem. Lett., 2009, 20, 288.

23. Rangnekar, D.; Kanetkar, V., Indian J. Fibre 
Text. Res., 1990, 15, 132.

24. Piorun, D.; Parusel, A.; Rechthaler, K.; Rotkiewicz, K.; Kohler, C., J. Photochem. Photobio. A Chem., 1999, 129, 33.

25. Butler, T.; Patel, N.; Robert, M.; Baynes, N., PCT Int. Appl. WO, 2005019159, 2006.

26. Gadhave, A. G.; Gaikar, R. B.; Kuchekar, S.
R.; Karale, B. K., J. Heterocycl. Chem., 2014, 51, 1849.

27. Gadhave, A. G.; Gaikar, R. B.; Kuchekar, S. R.; Karale, B. K., Indian J. Chem., 2015, 54B, 383.

28. Borhade, A. V.; Uphade, B. K.; Gadhave, A. G., Res. Chem. Int., 2015, 41, 1447.

29. Borhade, A. V.; Uphade, B. K.; Gadhave, A. G., Res. Chem. Int., 2016, 42, 6301. 